\title{
Gastric LTi cells promote lymphoid follicle formation but are limited by IRAK-M and do not alter microbial growth
}

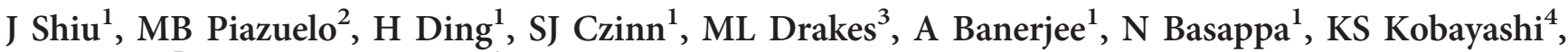 \\ WF Fricke ${ }^{5}$ and TG Blanchard ${ }^{1}$
}

Lymphoid tissue inducer (LTi) cells are activated by accessory cell IL-23, and promote lymphoid tissue genesis and antibacterial peptide production by the mucosal epithelium. We investigated the role of LTi cells in the gastric mucosa in the context of microbial infection. Mice deficient in IRAK-M, a negative regulator of TLR signaling, were investigated for increased LTi cell activity, and antibody mediated LTi cell depletion was used to analyze LTi cell dependent antimicrobial activity. H. pylori infected IRAK-M deficient mice developed increased gastric IL-17 and lymphoid follicles compared to wild type mice. LTi cells were present in naive and infected mice, with increased numbers in IRAK-M deficient mice by two weeks. Helicobacter and Candida infection of LTi cell depleted rag $1^{-1-}$ mice demonstrated LTi-dependent increases in calprotectin but not Reglll proteins. However, pathogen and commensal microbiota populations remained unchanged in the presence or absence of LTi cell function. These data demonstrate LTi cells are present in the stomach and promote lymphoid follicle formation in response to infection, but are limited by IRAK-M expression. Additionally, LTi cell mediated antimicrobial peptide production at the gastric epithelium is less efficacious at protecting against microbial pathogens than has been reported for other tissues.

\section{INTRODUCTION}

Lymphoid tissue inducer (LTi) cells promote secondary lymphoid tissue organogenesis during fetal development but are also important in the formation of ectopic lymphoid tissue in adults. ${ }^{1,2}$ LTi cells are a class of Group 3 innate lymphoid cells (ILC3s) characterized by expression of IL-17A and/or IL-22 and dependence on the transcription factor ROR $\gamma$ t for development and function. ${ }^{3,4}$ Murine LTi cells express CD4, CD90.2, CD117, and receptors for IL-1, IL-2, IL-7, and IL-23. ILC3 activation occurs largely through IL-23 binding, although TLR2 activation has been demonstrated to boost IL-22 production in human ILC3s when combined with IL-23. ${ }^{5}$ ILC3s also promote epithelial cell homeostasis and the production by epithelial cells of antimicrobial peptides through IL-17 and IL-22 secretion. These cells have largely been studied in the context of intestinal lymph tissue development and host resistance, but their role in the gastric mucosa has not been defined.

Helicobacter pylori (H. pylori) colonizes the stomach and is associated with peptic ulcer disease, gastric adenocarcinoma, and atrophic gastritis as well as gastric MALT lymphoma. ${ }^{6-8}$ The stomach generally lacks organized or diffuse lymphoid tissue but lymphoid follicles commonly develop in the gastric mucosa in response to $H$. pylori infection. These follicles have the potential to develop into low-grade gastric B-cell MALT lymphoma as $H$. pylori serves as an antigenic driver that can initiate the lymph-proliferative processes and the genesis of neoplastic clones. ${ }^{9,10}$ A small percentage of these indolent tumors undergo high-grade transformation with negative outcomes. The potential role of gastric LTi cells therefore has implications for pathogenesis as well as tissue neogenesis.

${ }^{1}$ Department of Pediatrics, University of Maryland School of Medicine, Baltimore, Maryland, USA. ${ }^{2}$ Department of Medicine, Vanderbilt University School of Medicine, Nashville, Tennessee, USA. ${ }^{3}$ Cardinal Bernardin Cancer Center, Loyola University Chicago, Maywood, Illinois, USA. ${ }^{4}$ Department of Microbial and Molecular Pathogenesis, Texas A \& M Health Science Center, Texas, USA and ${ }^{5}$ Institute for Genomic Sciences, University of Maryland, Baltimore, Maryland, USA. Correspondence: T Blanchard (tblanchard@peds.umaryland.edu)

Received 17 June 2014; accepted 8 December 2014; published online 21 January 2015. doi:10.1038/mi.2014.132 
We recently reported that IRAK-M, a negative regulator of TLR signaling, limits $H$. pylori induced dendritic cell maturation. ${ }^{11}$ In the absence of IRAK-M, stimulated dendritic cells expressed increased levels of MHC II and proinflammatory TNF $\alpha$ and MIP-2. We hypothesized LTi cells are present and active in the gastric mucosa, and that an IRAK-M deficiency would result in increased accessory cell activity leading to increased LTi cell mediated lymphoid follicle development during H. pylori infection. We further hypothesized that gastric LTi cells play an important role against pathogens as well as regulating commensal populations by promoting antimicrobial peptide production at the gastric epithelium. We now report LTi cells are present in the gastric mucosa, and that IRAK-M limits the development of $H$. pylori-associated lymphoid follicles. The activity of LTi cells in the stomach may be limited to lymphoid tissue neogenesis as $\mathrm{CD} 90.2^{+}$cell dependent antimicrobial peptide induction observed in two distinct models of gastric infection failed to alter the microbial burden of specific pathogens, or to alter the diversity or composition of the gastric microbiota. These results demonstrate that LTi cells are active in the gastric mucosa and are important for lymphoid tissue neogenesis in response to $H$. pylori infection. They also suggest that unlike previous descriptions of gut LTi cells, gastric LTi cell dependent antimicrobial activity has little impact on pathogens or on the commensal bacteria present at the gastric mucosa.

\section{RESULTS}

IRAK-M limits $H$. pylori-associated lymphoid follicle formation

WT and IRAK-M KO mice were infected with $H$. pylori to assess the overall impact of IRAK-M deficiency on $H$. pylori associated immunopathology. Subgroups of mice were harvested at four and 16 weeks post infection. No differences in the host response were observed at four weeks. Gastric inflammation was comparable between groups at 16 weeks although IRAK-M KO mice displayed increased acute inflammation in the corpus (Figure 1a). Bacterial loads were also comparable at 16 weeks although IRAK-M KO mice had several outliers $\left(2.62 \times 10^{7}\right.$ and $6.70 \times 10^{7}$ bacteria/gram tissue respectively; Figure 1b). IRAK-M KO mice however, develop increased H. pylori-specific serum IgG titers compared to WT mice by 16 weeks (Supplementary Figure 1a online). Similarly, there was a significant increase in lymphoid follicles in IRAK-M KO mice with structures apparent in almost all KO mice compared to less than $50 \%$ of WT mice, and a complete lack of follicles in naive mice of either strain $(P=0.041$; Figure 1c). Follicles were most common at the corpus-forestomach junction (Figure 1d). There was a 3.6 fold increase in the number of $\mathrm{CD}^{+}$cells in IRAK-M KO mice compared to WT mice by four weeks (9.28 vs 2.55 respectively; Supplementary Figure 1b) and by16 weeks, $46 \%$ of the lamina propria cells from IRAK-M KO mice were $\mathrm{CD} 4{ }^{+}$compared to $20.8 \%$ in the WT mice. PCR-based cytokine analysis demonstrated a significant increase in IL-17 in both WT and IRAK KO mice at 16 weeks with $\mathrm{KO}$ mice producing significantly greater amounts than WT mice
$(P=0.016$; Figure 1e). KO mice also had a notable but not significant increase in IL-23, and significantly less IL-10 than WT mice $(P=0.005)$. IL- 6 and IL-18 did not increase at either time point.

\section{Helicobacter associated lymphoid follicle formation is regulated independently of inflammation}

$H$. felis rapidly induces significant gastric inflammation within several weeks and $H$. felis infected WT mice demonstrated significant increases in IRAK-M expression in gastric tissue by 14 days post infection $(P=0.034$; data not shown). Additionally, similar to our previous in vitro study on $H$. pylori stimulation of bone marrow derived dendritic cells (BMDC), ${ }^{11}$ we demonstrated that $H$. felis antigen was comparable to $H$. pylori antigen in upregulating IRAK-M expression in BMDC by four hours post-stimulation as measured by semi quantitative RT-PCR (3.46 vs 3.25 fold, respectively, data not shown). Therefore, mice were infected with $\mathrm{H}$. felis for 28 days to investigate IRAK-M function in a model of more pronounced and rapid inflammation. Infection of WT and IRAK-M KO resulted in gastritis similar to what we observed at 16 weeks in our $\mathrm{H}$. pylori infection (Figure 2a). The bacterial load for WT and IRAK-M KO mice remained high with average counts of $7.99 \times 10^{9}$ and $1.13 \times 10^{10}$ respectively (Figure 2 b). IRAK-M KO mice however, developed significantly greater numbers of lymphoid follicles than WT counterparts $(P=0.0025)$. Similar to the $H$. pylori model described above, $100 \%$ of infected IRAKM KO mice developed lymphoid follicles compared to $50 \%$ of WT mice (Figure 2c). H. felis induced greater numbers of lymphoid follicles per mouse than $H$. pylori and they were present throughout the mucosa (Figure 2d).

TLR2 signaling down regulates Helicobacter associated gastritis as TLR2 KO mice develop significantly greater histologic gastritis than WT mice. ${ }^{12,13}$ Therefore we also investigated TLR2 KO mice to compare gastritis and lymphoid follicle development with IRAK-M KO mice. This information would allow us to determine if inflammation and lymphoid follicle development were independently regulated. TLR4 KO mice were included as TLR4 has also been shown to be involved in the host response to H. pylori. ${ }^{14}$ Chronic inflammation of the corpus in TLR2 $\mathrm{KO}$ mice was significantly increased compared to WT mice (Figure 3). TLR2 KO mice did develop increased numbers of lymphoid follicles compared to WT mice but these numbers were not statistically significant (data not shown). Unlike the H. pylori model described in Figure 1 above, when tissue was analyzed for cytokine expression there was no increase in either IL-17 or IL-23 for IRAK-M KO mice compared to WT mice (Figure 2e). Similarly, there was no decrease in IL-10 in the IRAK-M KO mice. The failure to detect these differences may be due to the degree of gastritis induced in both strains of mice in response to $H$. felis which involve IL-23 and IL-17 mediated pathways of inflammation. There was an overall increase in the percentage of $\mathrm{CD} 4^{+}$cells in IRAK-M KO mice of 4 fold compared to WT mice (5.91 vs 1.45 respectively; Figure 2f) and analysis of this $\mathrm{CD} 4{ }^{+}$cell fraction revealed greater increases in IL-17 expressing cells for IRAK-M KO mice 
a

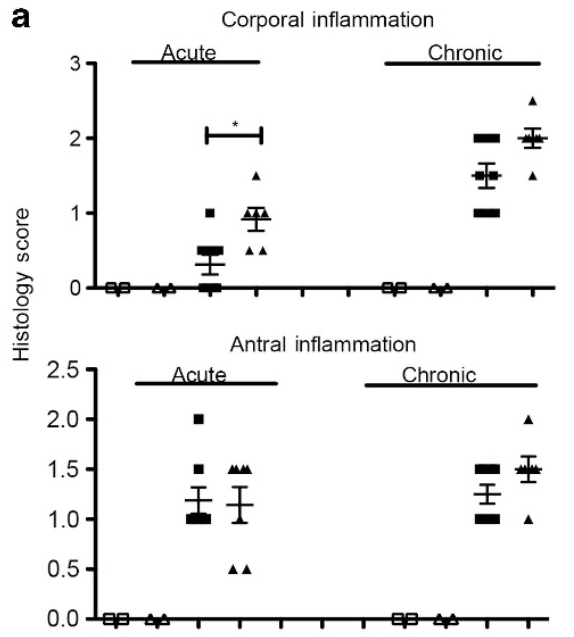

b $\left.3 \times 10^{8}\right] \quad$ Bacterial load

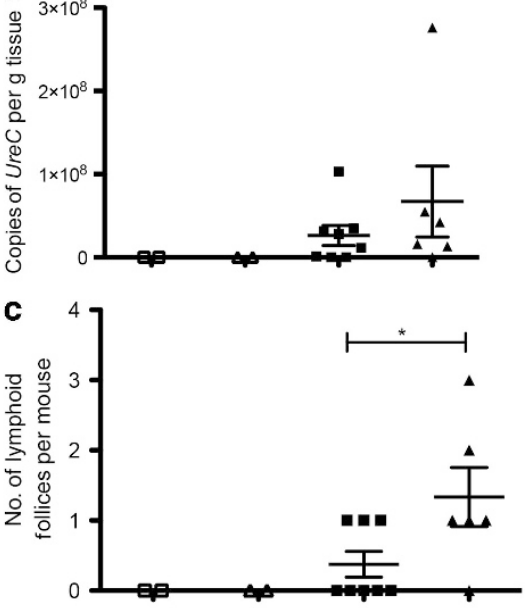

WT naive $\quad$ WT SS1+

$\Delta$ IRAK-M $\mathrm{M}^{-1-}$ naive $\triangle$ IRAK-M ${ }^{--} \mathrm{SS} 1+$
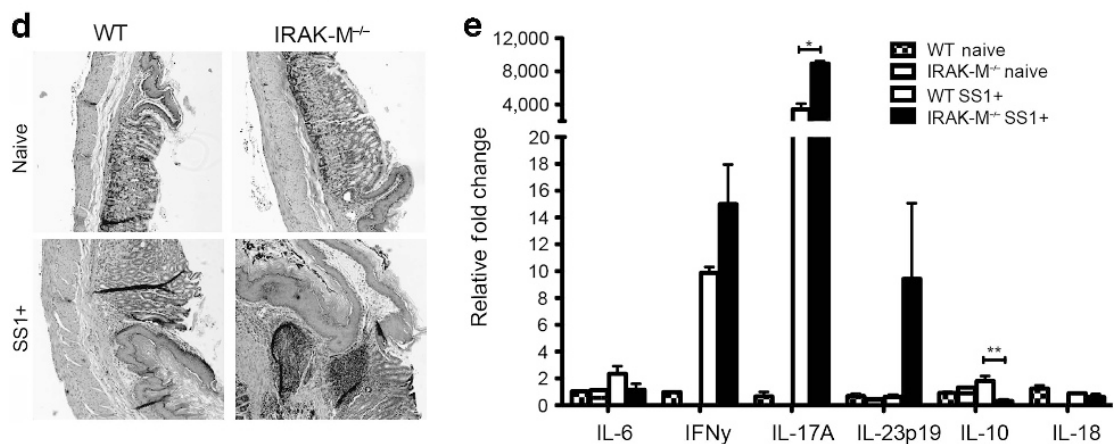

Figure 1 IRAK-M expression limits the development of $H$. pylori associated lymphoid follicles. WT and IRAK-M KO mice were infected with $H$. pylori for 16 weeks $(n \geq 6)$ (a) Acute and chronic inflammation were scored separately for the corpus and antrum on a scale of 0-3 ( \pm s.e.m.) (b) Bacterial load was determined by PCR quantification of ureC gene copy number per gram of stomach tissue ( \pm s.e.m.) (c) The number of lymphoid follicles present along the entire length of the gastric mucosa using histologic sections was determined ( \pm s.e.m.). (d) Representative H\&E stained stomach sections demonstrating the primary location of lymphoid follicles (100X). (e) Cytokine expression was determined by semi-quantitative PCR using RNA isolated from gastric tissue ( \pm s.e.m.). ${ }^{\star} P<0.05,{ }^{* *} P<0.01$.

which was consistent with $H$. pylori infection. Approximately $20 \%$ of $\mathrm{CD}^{+}$cells from IRAK-M KO mice expressed IL-17 compared to less than $3 \%$ for WT mice.

\section{CXCL13 levels are elevated in the absence of IRAK-M}

Proteins believed to play a role in lymphoid follicle development were measured in the gastric tissue of $H$. felis-infected mice to demonstrate LTi cell activity. No differences in LT $\alpha$, and LT $\beta$ were observed in $H$. felis-infected WT or IRAK-M KO mice compared to their respective controls, or to each other. (Figure 4a). Both CXCL13 and TNF $\alpha$ however, were significantly elevated in IRAK-M KO mice compared to WT mice $(P=0.0001$ and 0.011 respectively), although with respect to CXCL13 this difference may be due to an unexpected reduction in expression in infected WT mice. Subsequent analyses to assess these two cytokines over the course of a 28 day H. felis infection resulted in elevated CXCL13 expression in KO mice compared to WT mice from day three through 14 but these increases were not significant, and by day 28 were found to be equivalent (Figure $\mathbf{4 b}$ ). TNF $\alpha$ levels however were significantly greater by day 28 in $\mathrm{KO}$ mice compared to WT animals $(P=0.033)$. CXCL13 protein expression was also evaluated by immunohistochemistry given the recent demonstration of its importance in Helicobacter-associated lymphoid follicle formation(Figure 4c) ${ }^{15}$ Increased staining was evident in IRAK-M KO mice compared to infected WT mice. In situ hybridization revealed staining within lymphoid follicles in a reticular pattern that would suggest the involvement of fibroblast like cells, stromal cells, or follicular dendritic cells. The presence of any of these cell types is consistent with existing models of lymphoid follicle development.

\section{IL-17 ${ }^{+}$ILC are present in the gastric mucosa}

Group 3 ILCs such as LTi cells contribute to IL-17A production and induce lymphoid follicle development at mucosal surfaces. ${ }^{3,4}$ The impact of IRAK-M on lymphoid follicle 
a

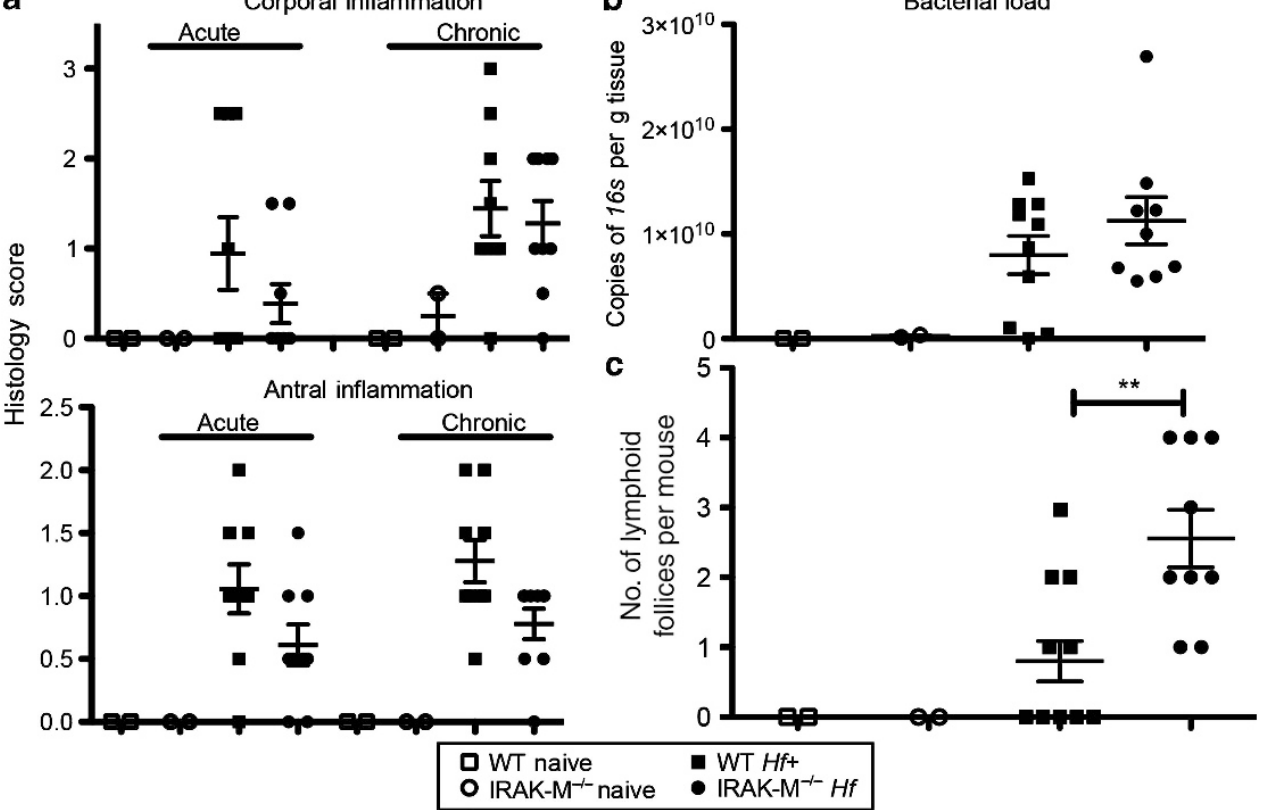

d

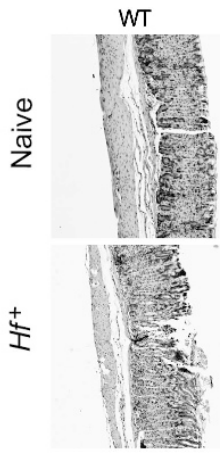

e
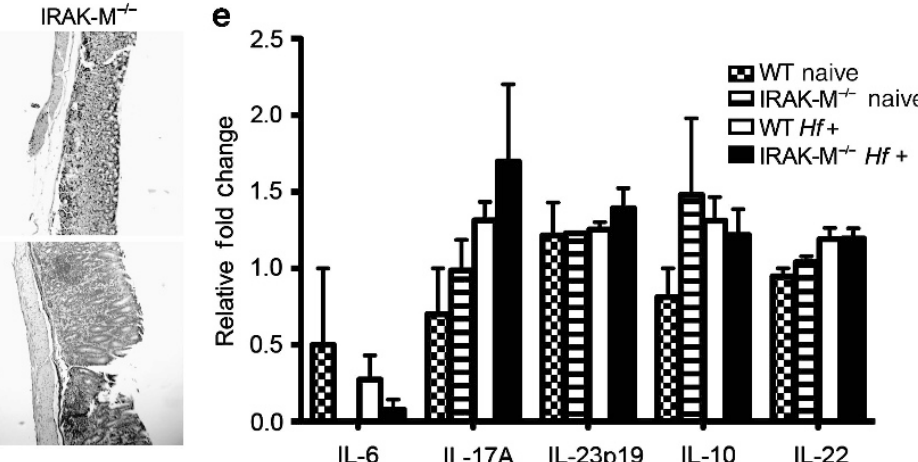

f
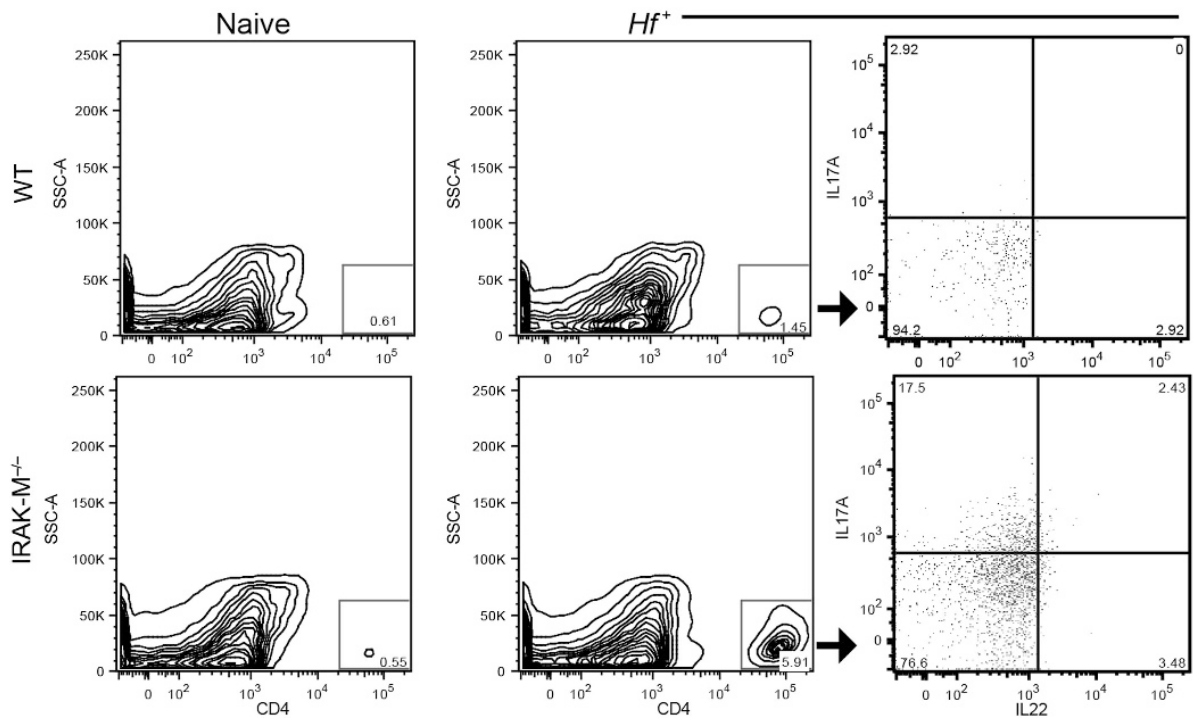

Figure 2 Lymphoid follicle development is increased in the absence of IRAK-M independently of inflammation. WT and IRAK-M KO mice were infected with $H$. felis for 28 days $(n \geq 9)$. (a) Acute and chronic inflammation were scored separately for the corpus and antrum on a scale of $0-3$ ( \pm s.e.m.).

(b) Bacterial load was determined by PCR quantification of 16S RNA gene copy number per gram of stomach tissue as described in Methods ( \pm s.d.).

(c) The number of lymphoid follicles present along the entire length of the gastric mucosa using histologic sections was determined ( \pm s.e.m.).

(d) Representative H\&E stained stomach sections demonstrating the primary location of lymphoid follicles (100X). (e) Cytokine expression was determined by semi-quantitative PCR using RNA isolated from gastric tissue ( \pm s.e.m.). (f) $\mathrm{CD}^{+}{ }^{+}$cells were isolated from the gastric lamina propria of naïve and $H$. felis-infected mice, stained for CD4 (contour plots), IL-17 and IL-22 (dot plots), and analyzed by flow cytometry. Data representative of three experiments. ${ }^{\star} P<0.05,{ }^{*} P<0.01$. 


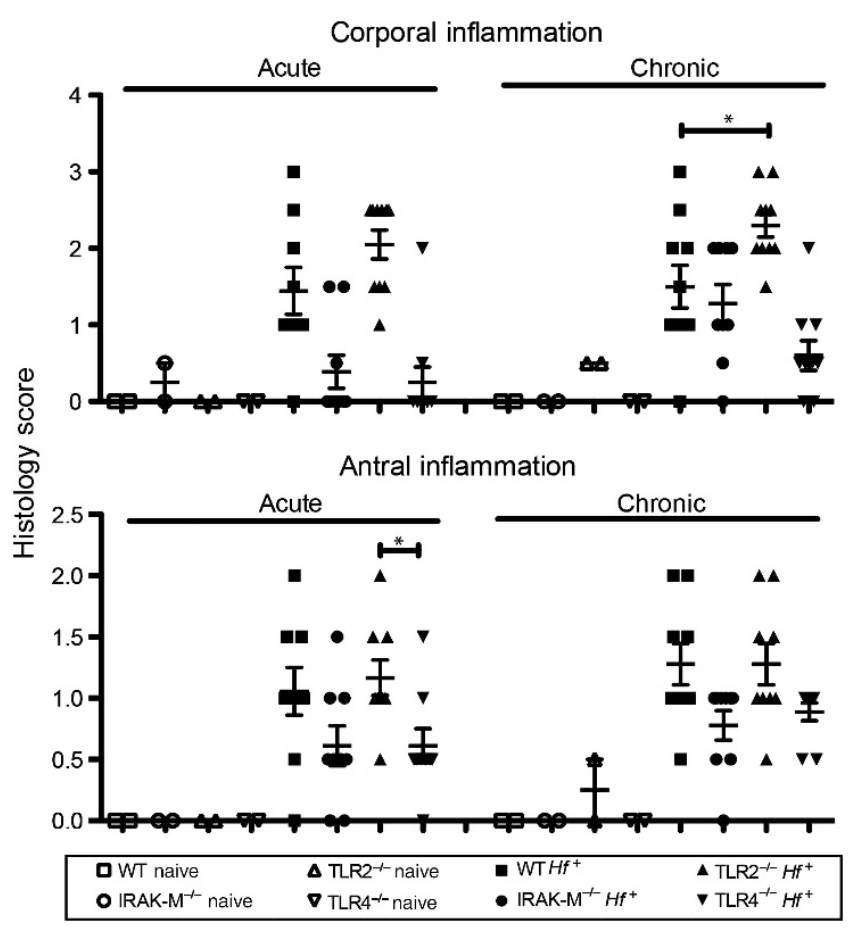

Figure 3 Absence of TLR2 or TLR4 does not result in overall changes in the character of $H$. felis induced gastritis. Groups of WT, IRAK-M KO, TLR2 KO and TLR4 KO mice were infected with $H$. felis for 28 days ( $n=8$ for infected groups). Longitudinal sections of the stomach encompassing the entire length of the greater curvature were evaluated for inflammation in the (a) corpus and (b) antrum using a scale of $0-3$. ${ }^{*} P<0.05$.

development described above led us to investigate whether ILCs are present in the gastric mucosa and how they may be affected during infection. Since there is no single ILC marker, and many ILC markers are shared with Th17 cells, ${ }^{16}$ we employed rag1 ${ }^{-1-}$ mice which lack B cells and Th cells to facilitate the isolation and analysis of ILCs. We created single cell suspensions from the stomachs of naive rag1-/- mice for analysis by flow cytometry. Using established markers (Lin ${ }^{-}$, CD90.2/Thy1.2 ${ }^{+}$, CD117/c-kit ${ }^{+}$and $\mathrm{CD}^{+}{ }^{+}$) for intestinal ILC populations, we identified a small population of $\mathrm{Lin}^{-}$(defined as $\mathrm{CD}^{-}$ CD $11 b^{-}$Ly-6G $^{-}$B220 ${ }^{-}$) CD90.2 ${ }^{+}$cells that express c-kit and CD4, which would fit the profile of murine CD4 ${ }^{+}$LTi cells (Figure 5a). Consistent with ILC3s, these ILCs expressed IL-22 and $\sim 20 \%$ of the IL-22 producers also produced IL-17A and stained positive for CD4 (Figure 5b). All of the cells in this population also expressed ROR $\gamma t$ (data not shown).In support of these results, we identified $\mathrm{CD} 4{ }^{+}$lymphoid cells in the gastric tissue of rag1 ${ }^{-1-}$ mice by immunohistochemistry with staining evident in the lamina propria of the gastric glands (Supplementary Figure 1c). Although gastric epithelial cells at the lumen and pits of the glands demonstrated high background staining, the morphology, location, and intensity of staining reveal ILCs. We also determined that IL-22 producing cells were present in lymphoreplete WT hosts and that the percentage of IL- $22^{+}$cells did not change with infection (data not shown). However, when we made a direct comparison of IRAK-M KO mice with WT mice infected with $H$. felis, we observed that only the IRAK-M KO mice demonstrated an increase of lineage negative, IL- $22^{+}$cells that were also $\mathrm{CD} 3$ deficient and $\mathrm{CD}^{+}$(Figure 5c). This change was evident by two weeks and by five weeks we were unable to detect these cells again. These data suggest a population of ILCs is present in the gastric mucosa, including a subgroup bearing resemblance to IL-17A and IL-22 producing LTi cells and that these cells increase in number following infection in the absence of IRAK-M. The low numbers of these cells however indicate that the predominant impact of IRAK-M is a decrease in LTi cells activity, not necessarily in LTi cell expansion. These data also indicate the difficulty in isolating these cells from gastric lamina propria as $\mathrm{CD}_{117^{+}} \mathrm{ROR}_{\gamma} \mathrm{t}^{+}$ cells were apparent within the lymphoid follicles of IRAK-M deficient mice four weeks post infection with $H$. felis (Figure 5d).

\section{Helicobacter infection induces LTi cell-dependent antibacterial peptide production}

LTi cells have been shown to induce epithelial cells to produce antimicrobial peptides such as RegIII and calprotectin. ${ }^{17}$ To investigate the antimicrobial role of LTi cells in the gastric mucosa, Thy1.2 positive cells were depleted from rag1 ${ }^{-1-}$ mice during infection with $\mathrm{H}$. felis. Although mesenchymal stromal cells also express CD90.2, these cells have not been reported to produce IL-22 or IL-17, or otherwise influence mucosal epithelial cells to produce antimicrobial peptides. ${ }^{18}$ LTi cells express several markers in common with Th cells and share activities such as IL-22 and IL-17 production with Th17 cells. Therefore, to study the role of ILCs without concern for compensatory Th17 function, we used $\operatorname{rag} 1^{-/}$mice which lack both $\mathrm{B}$ cells and T cells. No histologic differences were observed between infected and uninfected mice, regardless of ILC depletion. The bacterial load was comparable between groups (Figure 6a) $H$. felis infection did not increase expression of RegIII $\gamma$ (Figure 6b) above those observed in naive mice, but depletion of ILCs did significantly reduce levels of RegIII $\gamma$ $(P=0.0087)$ The peptides S100A8 and S100A9, which combine to form the antimicrobial heterodimeric protein calprotectin, were both significantly upregulated following infection $(P=0.0286$ and 0.0303 respectively) whereas depletion of ILCs reduced the levels of these peptides to those observed in naive mice (Figure 6c). Mice treated with isotype control antibody were similar to untreated infected mice. A separate evaluation of $\mathrm{ragl}^{-/-}$mice infected with $H$. pylori also demonstrated significant increases in S100A8 and S100A9 $(P<0.05$; Figure 6d). IL-22 and Csf2 were also evaluated in the tissue of the $H$. felis infected mice as these two cytokines have been shown to affect the expression of S100A8 and S100A9. IL-22 levels remained unchanged but Csf2 levels increased significantly in an ILC dependent manner $(P<0.05$; Figure 6e). Therefore, gastric ILCs are required for bacterial induced production of peptides which have been described in other models to have antimicrobial activity, although $H$. felis appears to be unaffected by these proteins. 
a

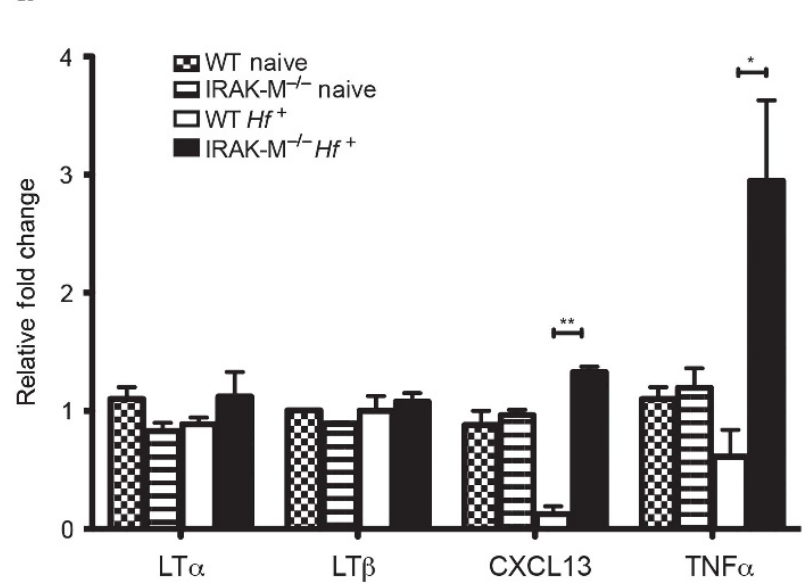

C
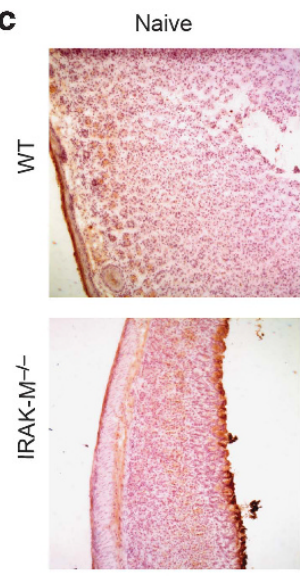

$\times 10$

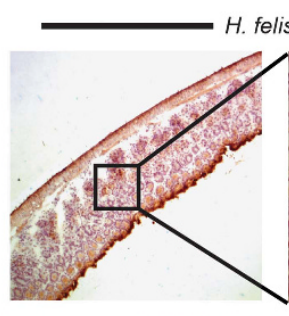

H. felis infected

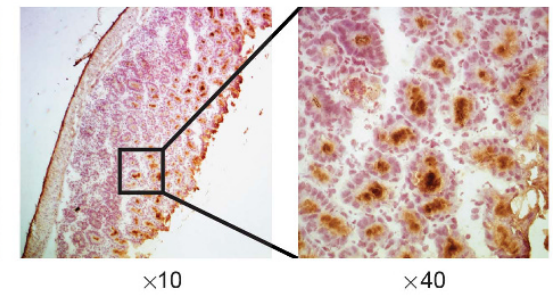

b

b $\quad$ CXCL13

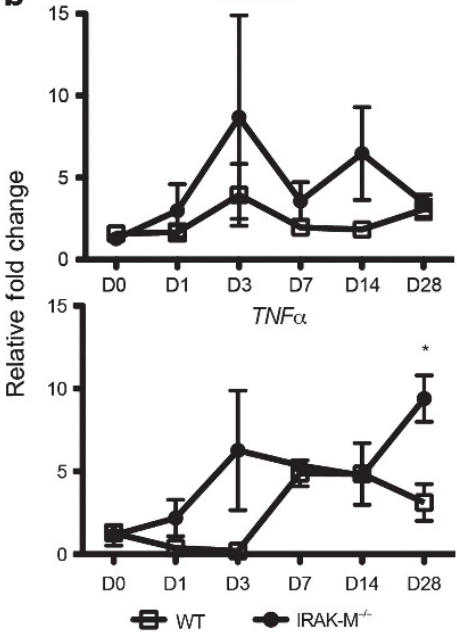

IRAK-M ${ }^{+-} \mathrm{Hf}^{+}$

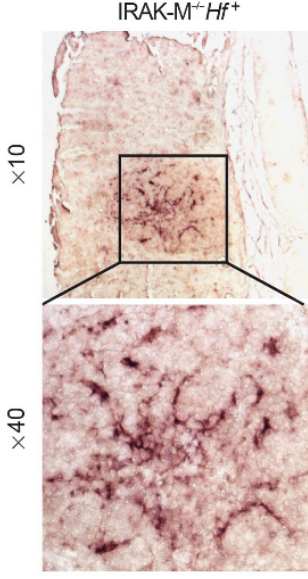

Figure 4 CXCL13 and TNF $\alpha$ levels are increased following Helicobacter infection in the absence of IRAK-M. (a) Gastric tissue from WT and IRAK-M KO mice infected with $H$. felis for four weeks were evaluated for cytokines associated with lymphoid neogenesis. Cytokine expression was determined by semi-quantitative PCR using RNA purified from gastric tissue ( $n=8$; \pm s.d.). (b) WT and IRAK-M KO mice infected with $H$. felis were evaluated for CXCL13 and TNF $\alpha$ over 28 days. Expression was determined by semi-quantitative PCR used purified RNA from gastric tissue $(n=3$ per time point per mouse strain; \pm s.e.m.). ${ }^{\star} P<0.05$. (c) Paraffin embedded tissues were stained for $\mathrm{CXCL} 13$ by immunohistochemistry (Left) or by in situ hybdridization with CXCL13 RNA-specific probes (right).

\section{LTi cell activity is not associated with significant changes in the gastric microbiome}

Antibacterial proteins induced indirectly by LTi cells are believed to help regulate bacterial growth at the mucosa as well as defend against pathogens. ${ }^{17}$ Therefore, microbial communities in LTi cell depleted animals were also assessed to determine if the lack of LTi cells would alter the composition of the microbiota. Population determinations were based on pyrosequencing of the bacterial 16S rRNA gene PCR amplicons. Species diversity was not significantly altered by the presence or absence of ILCs when evaluated by operational taxonomic units (OTUs) using rarefraction curves (Figure 7a). Similar results were achieved when comparing the relative microbiota compositions at the phylum level (Figure 7b). Hierarchical sample clustering based on microbiota compositions at the phylum level also failed to identify significant changes between groups (Figure 7c), as did analysis at the genus level (data not shown).
LTi cells do not promote eradication of Candida albicans from the gastric mucosa

The fungal pathogen $C$. albicans can colonize the murine stomach when mice are pretreated with cefoperazone. ${ }^{19}$ Gladiator et al. employed an anti-CD90.2 depletion model to demonstrated that ILC3s are protective against oropharyngeal candidiasis. ${ }^{20}$ We used the cefoperazone treatment protocol to infect the stomachs of $\operatorname{rag} 1^{-1-}$ mice with C. albicans in order to test a second gastric infection model for antimicrobial ILC3 activity. Groups of mice were treated with anti-CD90.2 or isotype control $\mathrm{mAb}$ as described above. Mice developed marked PMN infiltration predominantly in the corpus and corpus-forestomach junction with yeast evident in many samples (Figure 8a). Overall inflammation scores were not different between anti-CD90.2 and isotype control treated groups (data not shown). The calprotectin protein S100A9 was elevated in infected animals in an ILC-dependent manner $(P=0.029$; Figure 8b). The $C$. albicans burden in gastric tissue 
a

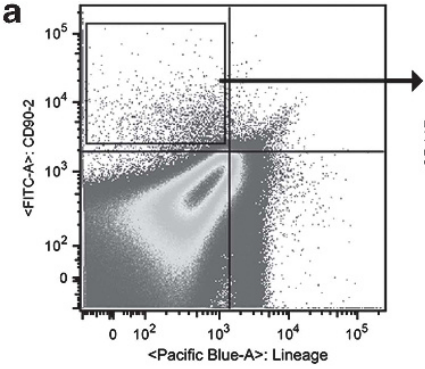

C
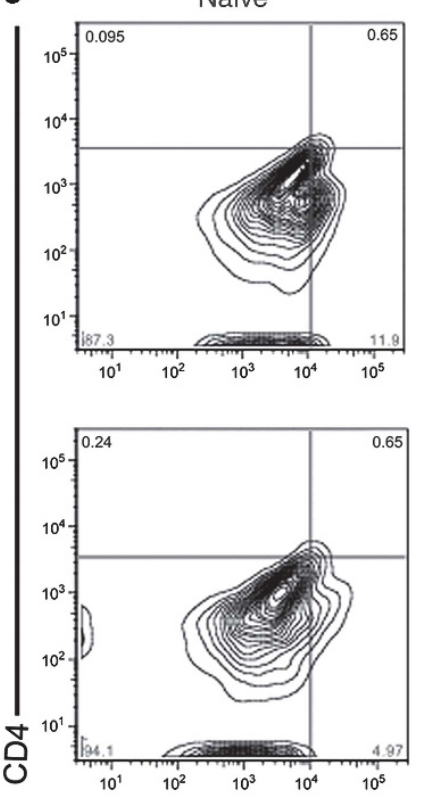

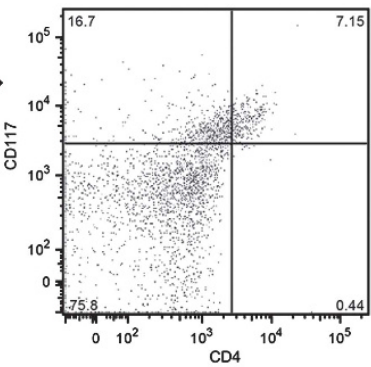

2 Weeks
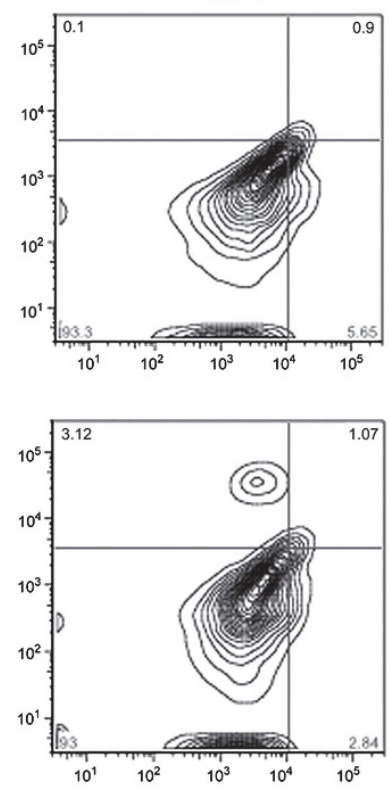

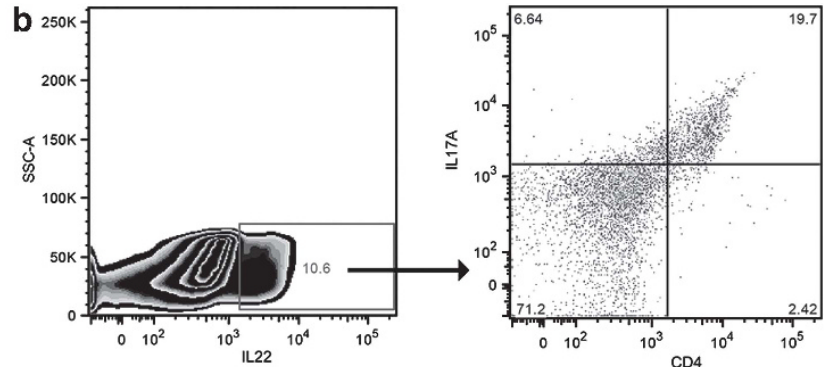

5 Weeks

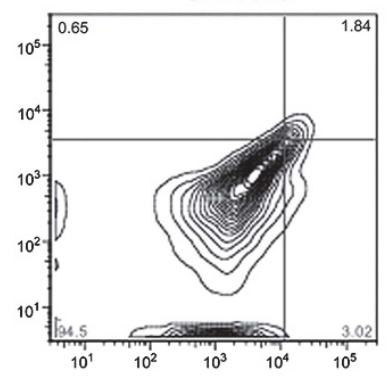

d
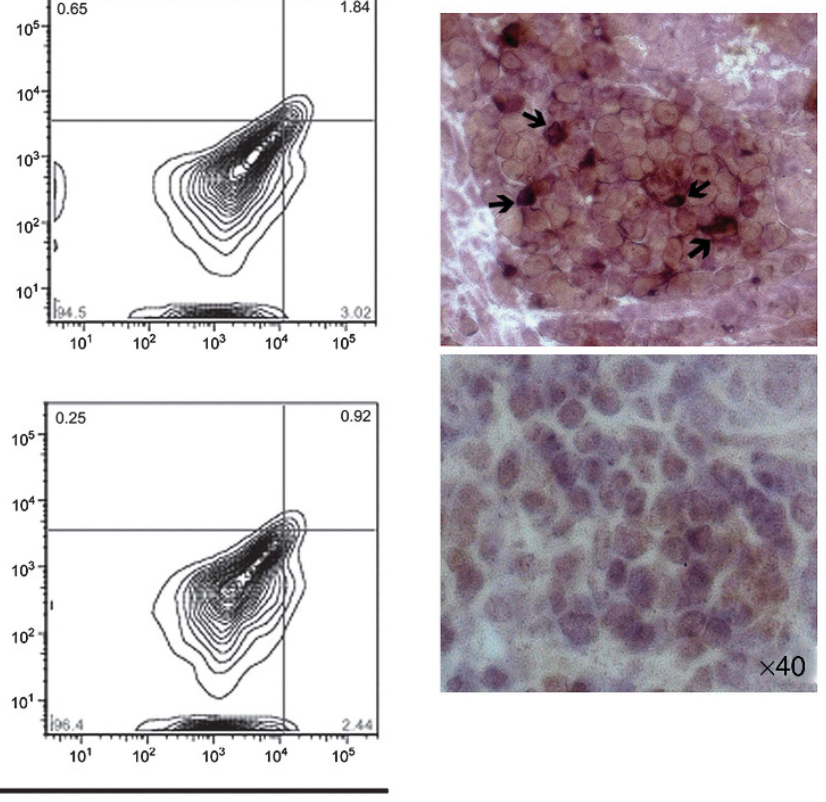

CD3

Figure 5 LTi cells are present in the gastric mucosa. (a) Lineage negative Thy1.2 + lymphocytes from the gastric mucosa were labeled with monoclonal antibodies for CD90.2, CD4 and CD117 (Table 2) and quantified by flow cytometry. (b) The Lin ${ }^{-}$Thy $1.2^{+}$ILC population was labeled by intracellular staining with antibodies for IL-22 and IL-17 and subpopulations were characterized by flow cytometry. (c) CD4 abd CD3 expression of single cell suspension of gastric lamina propria cells from $\mathrm{H}$. felis infected IRAK-M KO and WT mice gated for live cells and IL-22 expression. (d) ILCs prevalent in the lymphoid follicles of IRAK-M KO mice (top panel) based on dual positive immunostaining for nuclear expression of ROR $\gamma \mathrm{t}$ (purple with black arrows) and cellular expression of CD117 (brown). A serial section without antibody but subject to similar staining serves as a negative control (bottom panel). 400X.

however, as determined by PCR was comparable between groups and not different from infected mice without antibody treatment (Figure 8c).

\section{DISCUSSION}

The gastric mucosa normally lacks organized lymphoid tissue but gastric MALT B-cell lymphoma develops in response to H. pylori infection. ${ }^{21,22}$ Ectopic gastric lymphoid tissue resembles secondary lymphoid organs, but the signals that trigger ectopic lymphoid tissue formation in the context of chronic infection remain uncharacterized. We demonstrate that while IRAK-M has little impact on regulating inflammation or bacterial load, lack of IRAK-M leads to increased LTi cell activity, as demonstrated by increased numbers of LTi cells and significantly increased lymphoid follicle formation in the gastric lamina propria. These results suggest that IRAK-M normally suppresses signaling events that contribute to the formation of these structures. In support of the increased frequency of lymphoid follicles in infected IRAK-M deficient mice, we also observed higher antibody titers and $\mathrm{T}$ cell accumulation.

IRAK-M is a negative regulator of TLR signaling. ${ }^{23}$ It can mediate NF- $\kappa \mathrm{B}$ expression but can also results in the expression of inhibitory molecules such as A20, SOCS- 1 and SHIP- 1 and inhibition of translation of proinflammatory cytokines and chemokines. ${ }^{24}$ These mechanisms are consistent with our understanding of IRAK-M playing a role in maintaining immune homeostasis. ${ }^{25}$ A role for IRAK-M in limiting infection-associated lymphoid follicle development implicates TLRs. We did observe an increase in follicle formation in TLR2 KO mice that, while not significantly greater than WT mice, was not significantly lower than IRAK-M KO mice. This increase occurred in the absence of increased gastritis. Increased numbers of lymphoid follicles in TLR2 and IRAK-M KO mice without an increase in overall inflammation indicates that 


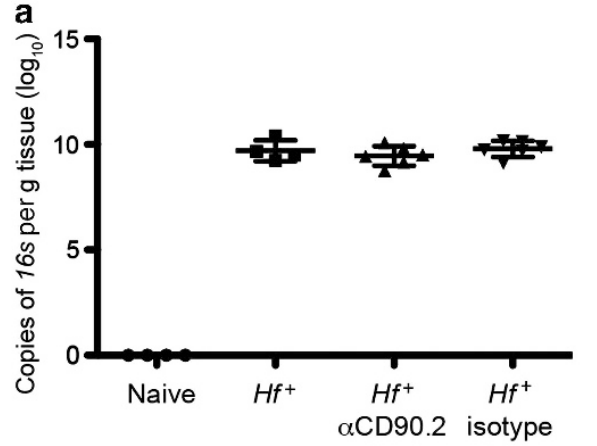

C

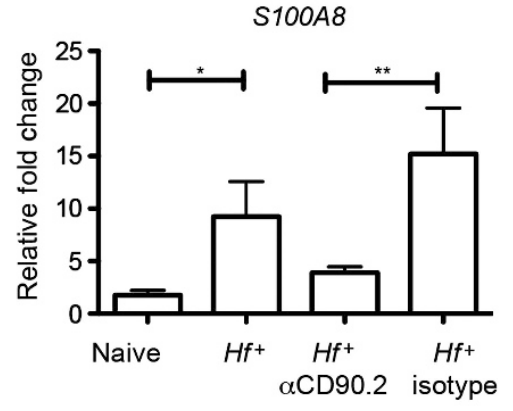

d

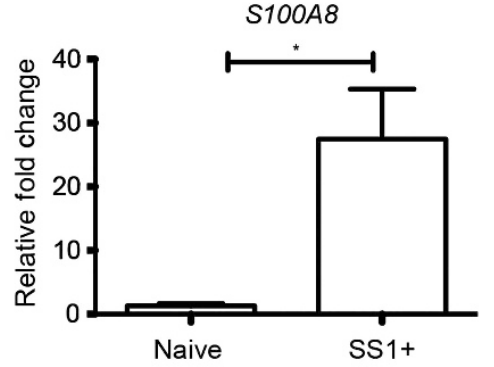

e

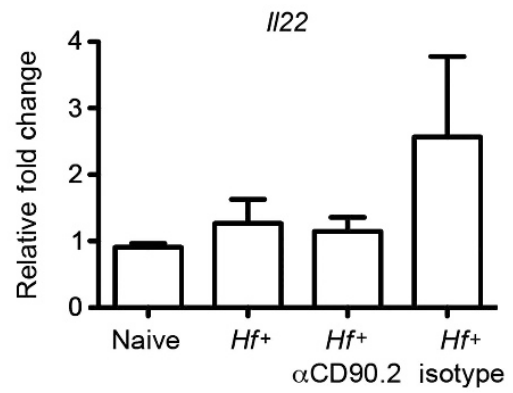

b

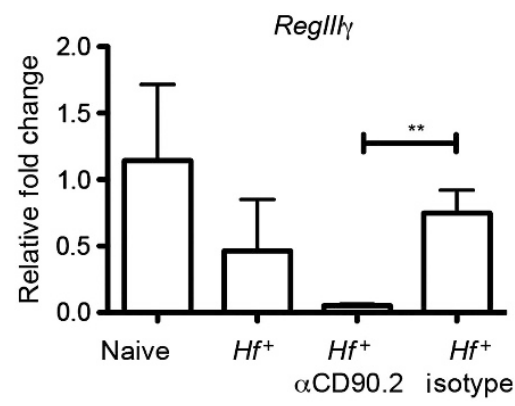

S100A9
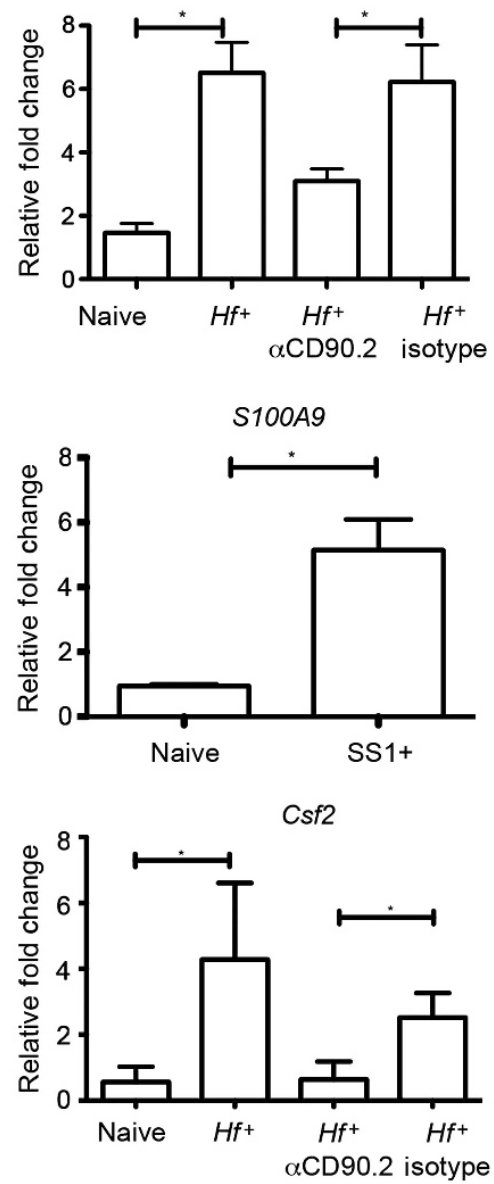

Figure 6 Helicobacter induces ILC dependent increases in calprotectin. (a) Groups of rag $1^{-1-}$ mice were infected with $H$. felis and treated with ILC-depleting antibody (anti-CD90.2) or an isotype matched control antibody. Bacterial load was determined by PCR quantification of 16S RNA gene copy number per gram of stomach tissue ( \pm s.d.). Host expression of (b) Reglll $\gamma$ and (c) calprotectin proteins S100A8 and S100A9 were determined by semi-quantitative PCR using RNA isolated from gastric tissue ( \pm s.e.m.). (d) Groups of rag $1^{-1-}$ mice were infected with $H$. pylori SS1 for 28 days and calprotectin protein levels were determined by semi-quantitative PCR ( \pm s.e.m.). (e) Cytokine expression was determined by semi-quantitative PCR using RNA isolated from the gastric tissue the groups described in (a) ( \pm s.e.m.).

infection induced lymphoid follicle formation operates independently of inflammation. Such activity is consistent with current models of LTi cell activity and we identified LTi cells in the gastric mucosa of naïve and infected mice by both flow cytometry and IHC. LTi cells are important initiators of lymphoid follicle development through LT $\alpha / \mathrm{LT} \beta$ expression which act on stromal cells to promote secondary lymphoid tissue development. ${ }^{26}$ We isolated gastric $\mathrm{CD} 4{ }^{+} \mathrm{IL} 22^{+}$cells from $\mathrm{rag} 1^{-l-}$ mice, and lineage negative, $\mathrm{CD} 3$ negative, $\mathrm{CD} 4{ }^{+} \mathrm{IL} 22^{+}$gastric cells from IRAK-M KO mice. We also detected $\mathrm{CD} 117^{+} \mathrm{ROR} \gamma \mathrm{t}^{+}$lymphoid cells in the lymphoid follicles of IRAK-M KO mice.

This increase in LTi cell activity is consistent with the significantly elevated levels of IL-23 we observed in our 
a

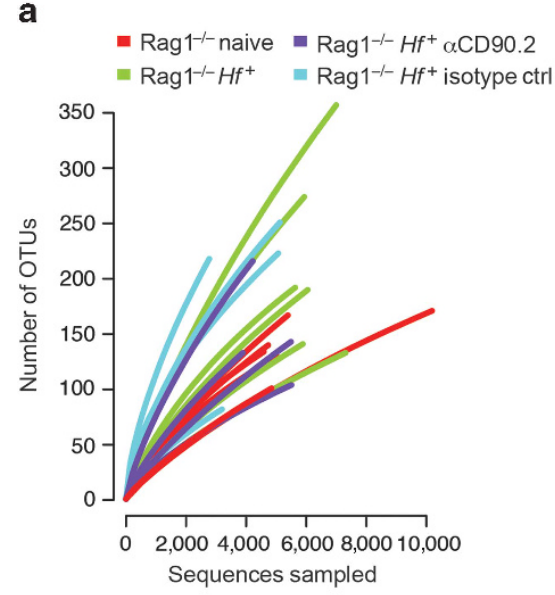

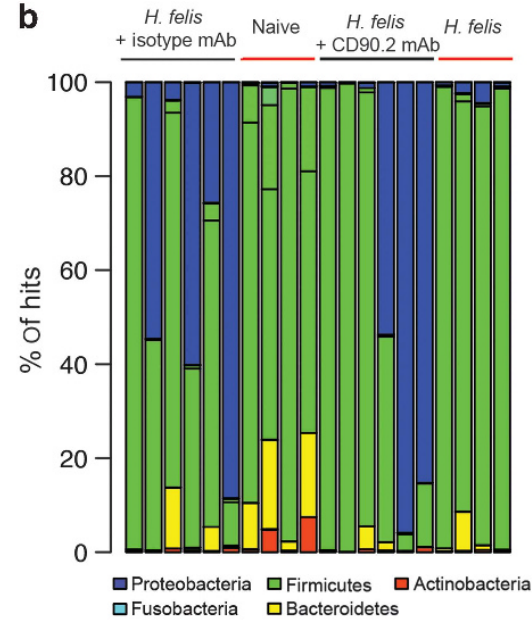

c

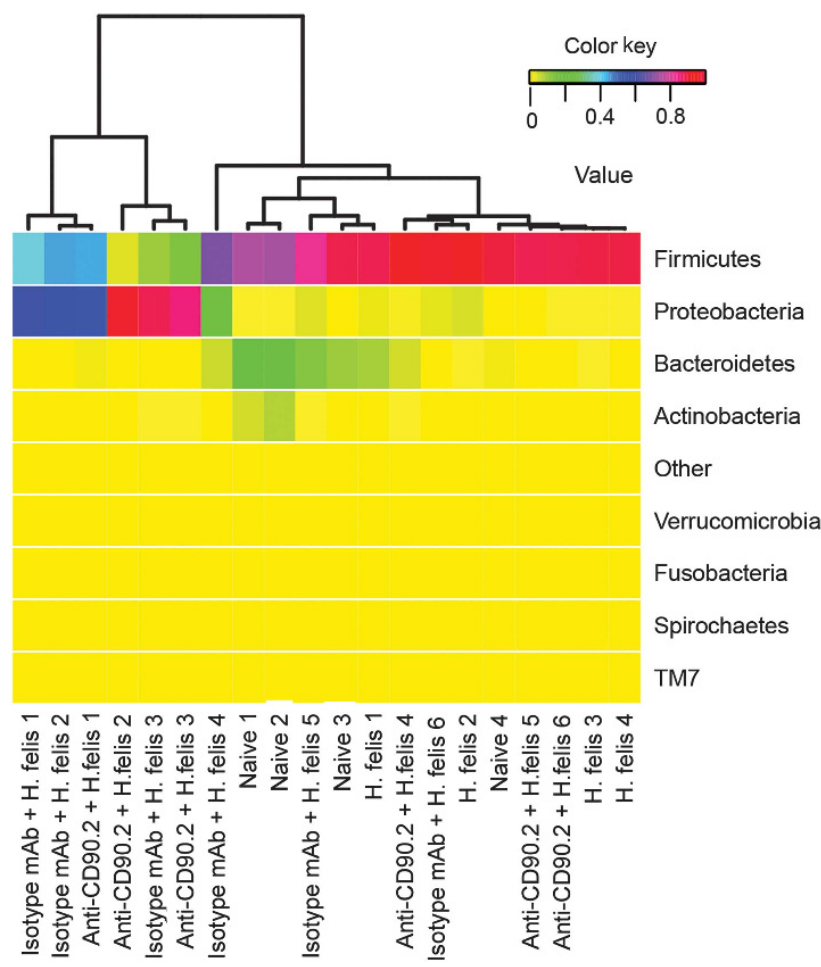

Figure 7 Gastric microbiota populations remain unchanged in the presence or absence of ILC-associated antimicrobial peptides. Groups of rag $1^{-1-}$ mice were infected with $\mathrm{H}$. felis and treated with ILC-depleting antibody or an isotype control antibody. (a) Microbiota rarefraction curves. (b) Relative gastric microbiota compositions at the phylum level. (c) Hierarchical sample clustering based on 16S rRNA microbiota compositions at the phylum level. Colors (yellow to red) show relative abundance per sample. Branches of the tree show sample similarities based on hierarchical clustering.

IRAK-M KO mice. The increased levels of CXCL13 and TNF $\alpha$ expression in H. felis-infected IRAK-M KO mice as well as the follicular staining pattern we achieved with anti-CXCL13 antibody are also consistent with increased lymphoid follicle formation. Mice deficient in CXCR5, the CXCL13 receptor, have decreased lymphoid follicle formation, as well as reduced IgG titers and IL-17A production, and Yamamoto et al. recently demonstrated that depletion of CXCL13 inhibits gastric lymphoid follicle formation in the $H$. suis infection mouse model. ${ }^{15}$
In light of the role that IRAK-M plays in limiting lymphoid follicle development, it may be significant that LTi cells have been demonstrated to express TLRs. We confirmed the expression of TLR2 and TLR4 on isolated gastric LTi cells in our model (data not shown). Crelin et al. employed expanded tonsil-derived ILC cell lines to demonstrate the potential of these cells to respond to TLR2 ligands, which resulted in the production of IL- 5 and IL-13 in addition to IL-22. ${ }^{5}$ The ability of LTi cells to respond directly to microbial antigens either alone or in combination with accessory cell cytokines suggests 

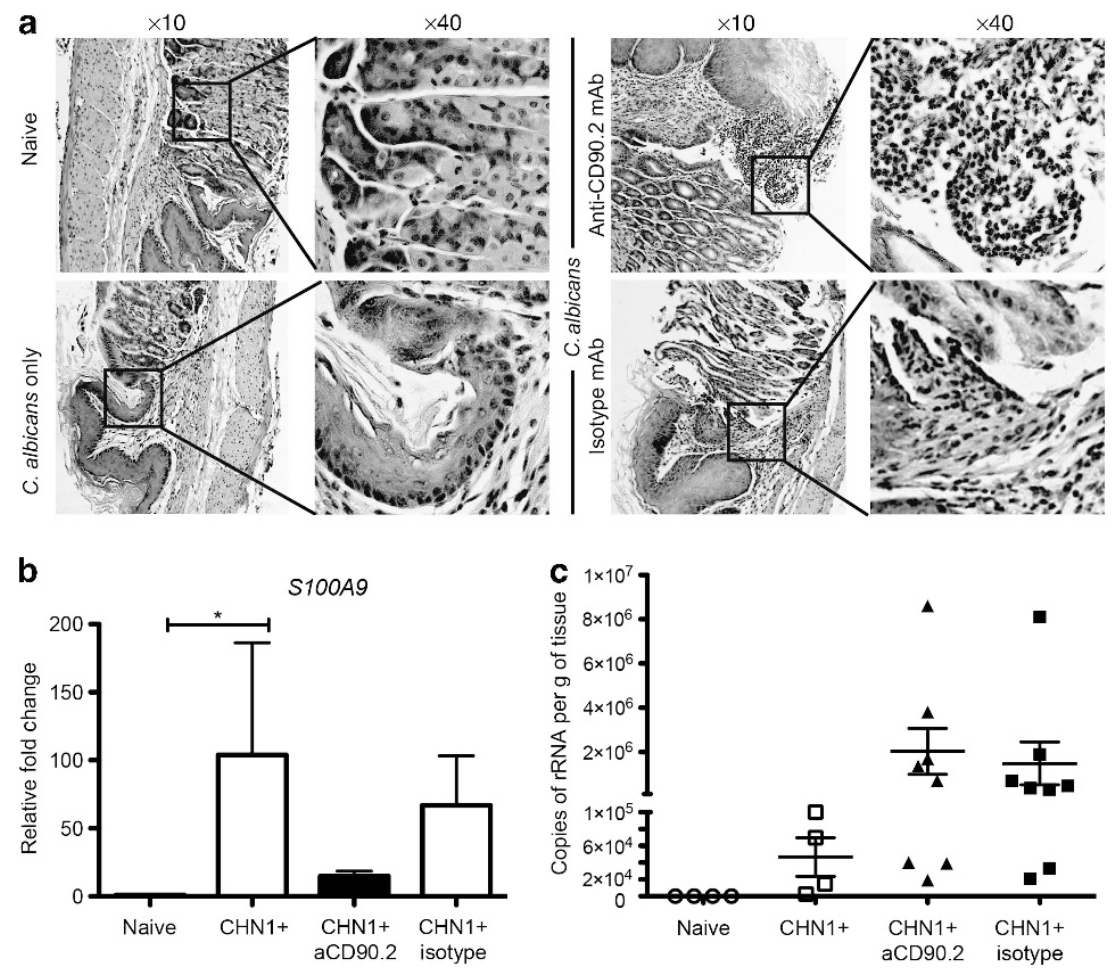

Figure 8 C. albicans infection of the gastric mucosa is resistant to ILC dependent increases in calprotectin. Groups of rag $1^{-1-}$ mice were infected with C. albicans and treated with ILC-depleting antibody (anti-CD90.2) or an isotype matched control antibody ( $n \geq 8$ ). (a) Representative H\&E stained stomach sections demonstrating the primary location and nature of lesions in response to infection. (b) Calprotectin subunit S100A9 levels were determined by semi-quantitative PCR on total RNA isolated from the gastric mucosa ( \pm s.e.m.). (c) Fungal load was determined by quantitative PCR of the ITS region of the rRNA gene of $C$. albicans and reported as copy number per gram of stomach tissue $\left( \pm\right.$ s.e.m.). ${ }^{\star} P<0.05$.

the increased lymphoid follicle numbers we observed in the absence of IRAK-M may be due in part to reduced IRAK-M activity in the LTi cells as well as the accessory cells. An Investigation into the expression and activity of IRAK-M in these cells is ongoing.

Finally, these data indicate LTi cell-dependent anti-microbial peptide production by epithelial cells varies from what has been described in the gut. Activated LTi cells produce IL-22 and IL-17 which influence antimicrobial activity at the epithelium. Whereas IL-22 induces RegIII and calprotectin peptides in response to bacterial pathogens, ${ }^{27-29}$ RegIII peptides were not induced following Helicobacter infection. Calprotectin however was upregulated by $H$. pylori, $H$. felis, and C. albicans in an LTi cell dependent manner. Additionally, whereas LTi production of IL-22, and subsequent induction of antimicrobial peptides, are important for limiting Citrobacter rodentium invasion in the mouse colon, ${ }^{30}$ and LTi cell activity has been demonstrated to be critical for controlling C. albicans infection in an oropharyngeal infection model, ${ }^{20}$ we found no changes in $H$. felis or C. albicans levels when LTi cells were depleted from rag $1^{-1-}$ mice throughout the course of infection. The diversity and relative representation of bacterial populations in the local gastric microbiota also remained unchanged by either increased calprotectin expression or depletion of LTi cells. These data indicate that the antimicrobial function of LTi cells may be limited in the stomach compared with other tissues owing to either the nature of the colonizing organisms, the tissue microenvironment, or distinctions in expression patterns of antimicrobial peptides.

\section{METHODS}

Mice. C57BL/6, TLR2 KO, TLR4 KO, and Rag1 KO mice were obtained from Jackson Laboratory (Bar Harbor, ME). IRAK-M KO mice derived on a C57BL/6 background were generated as previously described ${ }^{23}$ and bred at the University of Maryland School of Medicine (Baltimore, $\mathrm{MD})$. All mice were housed under pathogen-free conditions in microisolator cages at the University of Maryland Baltimore animal resource center.

Microbial strains and infection. H. pylori Sydney Strain 1 (SS1) and H. felis were grown on Columbia agar (Difco, Detroit, MI) with 7\% horse blood as previously described. ${ }^{11}$ Infections were by oral gavage $\left(1 \times 10^{7} \mathrm{CFU}\right.$ in $\left.0.5 \mathrm{ml}\right)$. H. pylori antigen for ELISA was prepared as previously described. ${ }^{11}$ Candida alibicans (C. albicans) CHNI strain was purchased from ATCC and grown on Sabouraud dextrose media. C. albicans infection was accomplished by pretreating mice with $0.5 \mathrm{mg} / \mathrm{ml}$ Cefoperazone (Sigma) in drinking water for 7 days prior to infection. ${ }^{19}$ Mice were challenged on day eight by oral gavage $\left(1 \times 10^{7}\right.$ CFU in $200 \mu \mathrm{l})$.

Microbial load determination. Helicobacter was quantified from total DNA extracted from a longitudinal strip of the stomach using DNeasy (Qiagen) with an additional $10 \mathrm{~min} .95^{\circ} \mathrm{C}$ incubation following Proteinase K digestion. PCR amplification was performed in triplicate with SYBR Green supermix (Fermentas, Glen Burnie, MD) using primers for ureC and comparison to a standard curve consisting of 
chromosomal DNA from $H$. pylori SS1. ${ }^{31} H$. felis was quantified in similar fashion using primers for the 16S RNA gene and a standard curve consisting of chromosomal $H$. felis DNA. ${ }^{32}$ For C. albicans quantification, total DNA was isolated from gastric strips using DNeasy (Qiagen) followed by ZR Fecal DNA Miniprep (Zymo Research). Quantitative PCR was performed as above using primers specific for the ITS region of the rRNA gene and a standard curve consisting of chromosomal DNA purified from C. albicans. ${ }^{33}$

Histopathology. A longitudinal strip encompassing the entire length of the stomach from the forestomach to the duodenum was fixed in $10 \%$ buffered formalin, and paraffin embedded. H\&E-stained sections were graded in a blinded fashion by a pathologist (MBP). The corpus and antrum were graded separately for acute (polymorphonuclear neutrophils) and chronic (lymphocytes, plasma cells and macrophages) inflammation using a $0-3$ scale. Lymphoid follicles were counted from the squamocolumnar junction to the pyloroduodenal junction.

Serum IgG ELISA. Plates were coated with $100 \mu \mathrm{l}$. pylori antigen $(10 \mu \mathrm{g} / \mathrm{ml})$ at $4{ }^{\circ} \mathrm{C}$. Wells were washed prior to each step three times with PBS/0.05\% Tween-20. Wells were blocked with $200 \mu \mathrm{l} \%$ BSA in PBS for $2 \mathrm{~h}$ and incubated one hour with $100 \mu \mathrm{l}$ mouse sera prepared in serial half log dilutions. Anti-H. pylori IgG was detected by incubation for one hour with $100 \mu$ l horseradish peroxidase conjugated goat antimouse antibody (Southern Biotechnology Assoc., Inc, Birmingham, AL) followed by TMB substrate (KPL, Gaithersburg, MD). End-point titers were defined as the highest dilution of sample to generate a signal at least $0.05 \mathrm{OD}$ units above the conjugate control.

Antibody-mediated depletion of ILC. LTi cell depletion was performed in rag1 ${ }^{-1-}$ mice by injection with $250 \mu \mathrm{g}$ anti-CD $90.2 \mathrm{mAb}$ (Clone $30 \mathrm{H} 12$ ) or isotype control $\mathrm{mAb}$ (clone LTF-2)(BioXCell, West Lebanon, NH) i.p every 3 days as previously reported. ${ }^{34}$ Antibody treatment was initiated two days prior to infection and maintained for 20 days for $H$. felis infection, or eight days for C. albicans infection.

In situ hybridization. CXCL13 cDNA template was amplified with primers CXCL13-FP (5' AGGTTGAACTCCCCTCCAG-3') and CXCL13-rP2 (5'-GGTGCAGGTGTGCTTTTG). RNA probes were transcribed and labeled with digoxigenin from template DNA using the DIG RNA Labeling Mix (Roche, Indianapolis, IN). Deparaffinized $5 \mu \mathrm{m}$ sections were pre-fixed in $4 \%$ paraformaldehyde (PFA), washed $3 \mathrm{x}$ in PBS and incubated in $20 \mu \mathrm{g} / \mathrm{ml}$ proteinase $\mathrm{K}$ for $20 \mathrm{~min}$. Sections were refixed with $4 \%$ PFA for $5 \mathrm{~min}$, washed $3 \mathrm{x}$ in PBS, $1 \mathrm{x}$ in $0.1 \mathrm{M}$ Triethanolamine, and then acetylated with $0.25 \%$ acetic anhydride for $10 \mathrm{~min}$. A total of 250-300 ng per slide was hybridized in Hybridization Solution (Sigma-Aldrich) with 50\% formamide and baker's yeast tRNA (Sigma-Aldrich) at $67^{\circ} \mathrm{C}$ in a humidifying chamber. Slides were washed in $5 \mathrm{X}$ saline-sodium Citrate (SSC) for $5 \mathrm{~min}$, twice in $0.2 \mathrm{X}$ SSC for $30 \mathrm{~min}$ at $72{ }^{\circ} \mathrm{C}$, then in $0.2 \mathrm{X} \mathrm{SSC}$ for $5 \mathrm{~min}$ at room temperature. Slides were blocked in $10 \%$ horse serum with $0.1 \mathrm{M}$ Tris/ $0.15 \mathrm{M} \mathrm{NaCl}$ for $2 \mathrm{~h}$ at room temperature. Anti-digoxigenin antibody (Roche Applied Science) diluted 1:2000 in blocking solution was added and incubated overnight at $4{ }^{\circ} \mathrm{C}$. Unbound antibodies were removed by washing four times in $0.1 \mathrm{M}$ Tris $/ 0.15 \mathrm{M} \mathrm{NaCl}$ for $5 \mathrm{~min}$. Slides were then washed in alkaline phosphatase buffer ( $100 \mathrm{~mm}$ Tris [pH 9.5]/ $100 \mathrm{~nm} \mathrm{NaCl}$ ) for $10 \mathrm{~min}$ and developed overnight in nitro blue tetrazolium (NBT)/5-bromo-4-chloro-3-indolyl phosphate (BCIP) (Roche) until staining was satisfactory. Slides were washed in $\mathrm{dH}_{2} \mathrm{O}$ three times for $5 \mathrm{~min}$, fixed in 4\% PFA for $5 \mathrm{~min}$, then washed three times in $\mathrm{dH}_{2} \mathrm{O}$ for $5 \mathrm{~min}$.

Immunohistochemistry. Mouse stomach tissue from control and experimental groups were paraffin embedded, and 4 um sections on slides were deparaffinized and boiled for 5 min under pressure in an antigen retrieval agent, Reveal (Biocare Medical, Concord, CA). Endogenous hydrogen peroxide was blocked with $0.4 \%$ hydrogen peroxide in DPBS for $20 \mathrm{~min}$. Slides were stained by first blocking with
$10 \%$ normal goat serum followed by Avidin and Biotin blocking reagents (SP2001, Vector Laboratories). Sections were incubated overnight with primary antibodies for CD4 (H-370; sc-7219, Santa Cruz Biotech) in 5\% blocking serum. Negative control sections were incubated in blocking serum only. Sections were developed using the rabbit ABC Staining System (sc-2018, Santa Cruz) and the chromogen diaminodenzidine (DAB, brown, from sc-2018kit). Tissue was counterstained with hematoxylin. Double staining for CD117 and ROR $\gamma$ t was performed by blocking with $2.5 \%$ horse serum and using an initial overnight stain with anti-ROR $\gamma \mathrm{t}$ antibody (OAEB00974, Aviva Systems Biology Corp, San Diego) followed by ImmPRESS regents for goat antibody (MP7405, Vector Laboratories, Burlingame, CA) and development with purple ImmPACT VIP HRP substrate (Vector). Sections were then stained overnight with anti-CD117 (AF1356, R\&D Systems) and goat ImmPRESS reagents followed by brown ImmPACT DAB HRP substrate (Vector).

Quantitative real-time PCR for cytokines and antimicrobial peptides. RNA was isolated from longitudinal strips of each stomach using RNeasy (Qiagen) and converted to cDNA using the Quantitect reverse transcription kit (Qiagen). PCR amplification was performed with SYBR Green supermix (Fermentas, Glen Burnie, MD). Reactions were performed in $25 \mu \mathrm{l}$ total volume including $0.8 \mu \mathrm{M}$ of each primer, and $1 \mu \mathrm{lcDNA}$. Primer sequences are listed in Table 1. Relative gene expression changes were calculated using the $2^{-\Delta \Delta \mathrm{CT}}$ method, and expression normalization was accomplished using the GAPDH housekeeping gene.

Flow cytometry. Gastric mucosa was collected by scraping using a glass slide and then strained (100 $\mu \mathrm{m}$ nylon mesh). Tissue was digested $1 \mathrm{~h}$ at $37^{\circ} \mathrm{C}$ in a PBS containing $1 \mathrm{mmol} / \mathrm{l}$ dithithreitol, $2 \mathrm{~mm}$ EDTA, HEPES, $10 \%$ FBS, and $100 \mathrm{U} / \mathrm{ml}$ of collagenase type VIII from Clostridium histolyticum. Cells were filtered (70 $\mu \mathrm{m}$ nylon mesh) and $\mathrm{CD}^{+}{ }^{+}$cells isolated using the MagCellect mouse $\mathrm{CD} 4{ }^{+} \mathrm{T}$ cell isolation kit (R\&D Systems, Minneapolis, MN). Cells were prepared with $5 \% \mathrm{FBS}$ and $5 \mu \mathrm{g} / \mathrm{ml} \mathrm{Fc}$ Block (eBioscience) at $4{ }^{\circ} \mathrm{C}$ for $5-10 \mathrm{~min}$. Labeled antibodies (Table 2 ) were added ( $1 \mu \mathrm{l} /$ million cells) for $30 \mathrm{~min}$ at $4{ }^{\circ} \mathrm{C}$ in the dark. Cells were washed twice with $5 \%$ FBS. For intracellular staining, cells were fixed and permeabilized in $1 \mathrm{ml}$ of cytofix/cytoperm solution for $20-30 \mathrm{~min}$ at $4{ }^{\circ} \mathrm{C}$ protected from light (BD Biosciences, San Jose, CA). Cells were then washed twice in $1 \mathrm{X}$ Perm Wash Buffer (BD Biosciences) and staining was performed in $100 \mu \mathrm{l}$ Perm Wash Buffer with labeled antibodies as above. Cells were washed and suspended in $350 \mu \mathrm{l}$ and analyzed using a LSRII flow cytometer (BD Biosciences) gated on live cells based on forward and side scatter, or using a live/dead fixable marker (LIVE/DEAD Fixable Dead Cell Stain Kit; Invitrogen). Data were analyzed using FlowJo7 software (Tree Star). Median fluorescence intensity was calculated as the difference between stimulated samples and unstimulated controls.

Gastric microbiota analysis. Tissue preparation and microbiota analysis were performed as previously described. ${ }^{35}$ Briefly, tissue was lysed at $37^{\circ} \mathrm{C}$ in a cocktail of lysozyme, mutanolysin and lysostaphin, followed by digestion at $56^{\circ} \mathrm{C}$ with a solution of Proteinase K, $10 \%$ SDS, and RNase. Cells were disrupted by bead beating in tubes with Lysing Matrix B (MP Biomedicals, Solon, OH) in a FastPrep-24. The lysate was processed using the SR Fecal DNA mini-prep kit (Zymo, Irvine, CA). Samples were eluted in $100 \mu \mathrm{l}$ ultrapure H2O. $50 \mathrm{ng}$ of DNA template was used for amplification of 16sRNA genes. Sequences were then binned, trimmed and filtered with QIIME. Chimeric clusters were removed with UCHIME. 16S rRNA sequence reads were processed with CloVR using the automated CloVR-16S pipeline. Reads were clustered into operational taxonomic units (OTUs) using a similarity threshold of $95 \%$. Rarefraction curves were calculated based on OTU counts using the rarefraction.single routine of the Mothur package. Hierarchical clustering was performed using R, a statistical analysis package. 
Table 1 Oligonucleotide primers for quantitative PCR

\begin{tabular}{|c|c|c|}
\hline Gene & Forward primer $\left(5^{\prime}-3^{\prime}\right)$ & Reverse primer $\left(5^{\prime}-3^{\prime \prime}\right)$ \\
\hline Csf2 & TGGAAGCATGTAGAGGCCATCA & GCGCCCTTGAGTTGGTGAAAT \\
\hline Gapdh & CCAGGTTGTCTCCTGCGACTT & CCTGTTGCTGTAGCCGTATTCA \\
\hline $\begin{array}{c}\text { Helicobacter } \\
16 S \text { rRNA }\end{array}$ & TCGGAATCACTGGGCGTAA & TTCTATGGTTAAGCCATAGGATITCAC \\
\hline Ifn $\gamma$ & CATGGCTGTTCTGGCTGTTACTG & GTTGCTGATGGCCTGATTGTCTIT \\
\hline 116 & ACAAGTCGGAGGCTTAATTACACAT & TTGCCATTGCACAACTCTITC \\
\hline $1 / 18$ & ACTGTACAACCGCAGTAATAC & AGTGAACATTACAGATTTATCCC \\
\hline II23p19 & TGCTGGATTGCAGAGCAGTTAA & GCATGCAGAATTCCGAAGA \\
\hline Irakm & TGAGCAACGGGACGCTIT & GATTCGAACGTGCCAGGAA \\
\hline$L t \alpha$ & CCAGGACAGCCCATCCACT & GTACCCAACAAGGTGAGCAGC \\
\hline$L t \beta$ & ACCTCATAGGCGCTTGGATG & ACGCTTCTTCTTGGCTCGC \\
\hline RegIII $\gamma$ & ATGGCTCCTATTGCTATGCC & GATGTCCTGAGGGCCTCTT \\
\hline Reglll $\beta$ & ATGGCTCCTACTGCTATGCC & GTGTCCTCCAGGCCTCTIT \\
\hline S100A8 & TGTCCTCAGTTTGTGCAGAATATAAA & TCACCATCGCAAGGAACTCC \\
\hline S100A9 & GGTGGAAGCACAGTTGGCA & GTGTCCAGGTCCTCCATGATG \\
\hline Tnfo & TCCCAGGTTCTCTTCAAGGGA & GGTGAGGAGCACGTAGTCGG \\
\hline
\end{tabular}

Table 2 Labeled antibodies for flow cytometry

\begin{tabular}{|c|c|c|c|c|}
\hline LINEAGE Neg & Pac-Blue & Multiple & Rat IgG2b/Rat IgG2a & BioLegend \\
\hline CD4 & APC & GK1.5 & Rat lgG2b, $\kappa$ & eBioscience \\
\hline CD117 (c-Kit) & $\mathrm{PE}$ & 2B8 & Rat lgG2b, $\kappa$ & eBioscience \\
\hline CD90.2 (Thy1.2) & FITC & $30-\mathrm{H} 12$ & Rat lgG2b, $\kappa$ & eBioscience \\
\hline IL-22 & $\mathrm{PE}$ & Polyclonal & Goat lgG & eBioscience \\
\hline$\underline{\mathrm{ROR} \gamma(\mathrm{t})}$ & PerCP & $\mathrm{B} 2 \mathrm{D}$ & Rat lgG1, $\kappa$ & eBioscience \\
\hline
\end{tabular}

Statistical analysis. Statistical analysis was performed using GraphPad Prism for Macintosh 5.0c (GraphPad Software, San Diego, CA). The Mann-Whitney test was used for analysis of statistical significance and a $P$-value $<0.05$ was considered significant.

SUPPLEMENTARY MATERIAL is linked to the online version of the paper at http://www.nature.com/mi

\section{ACKNOWLEDGMENTS}

This work was supported by grants from the NIH/NIDDK (SJC;RO1-DK46461), NIAID (TGB;RO1-AI-055710), and the Canadian Institutes of Health Research JS;DFSA-236840).

\section{DISCLOSURE}

The authors declare no conflict of interest.

c) 2015 Society for Mucosal Immunology

\section{REFERENCES}

1. Mebius, R.E., Rennert, P. \& Weissman, I.L. Developing lymph nodes collect CD4 + CD3- LTbeta + cells that can differentiate to APC, NK cells, and follicular cells but not T or B cells. Immunity 7, 493-504 (1997).

2. Yoshida, H. et al. IL-7 receptor alpha + CD3(-) cells in the embryonic intestine induces the organizing center of Peyer's patches. International immunology 11, 643-655 (1999). 
3. Spits, H. \& Cupedo, T. Innate lymphoid cells: emerging insights in development, lineage relationships, and function. Annual review of immunology 30, 647-675 (2012).

4. Walker, J.A., Barlow, J.L. \& McKenzie, A.N. Innate lymphoid cells-how did we miss them? Nature reviews. Immunology 13, 75-87 (2013).

5. Crellin, N.K. etal. Regulation of cytokine secretion in human CD127(+) LTilike innate lymphoid cells by Toll-like receptor 2. Immunity 33, 752-764 (2010).

6. $\mathrm{NIH}$ Consensus Conference. Helicobacter pylori in peptic ulcer disease. Journal of the American Medical Association 272, 65-69 (1994).

7. World Health Organization. In Schistosomes, Liver Flukes and Helicobacter pylori Vol. 61 IARC monographs on the evaluation of carcinogenic risks to humans, 177-241 (International Agency for Research on Cancer 1994, Lyon, France).

8. Wotherspoon, A.C., Ortiz-Hidalgo, C., Falzon, M.R. \& Isaacson, P.G. Helicobacter pylori-associated gastritis and primary B-cell gastric lymphoma. Lancet 338, 1175-1176 (1991).

9. Craig, V.J. et al. Gastric MALT lymphoma B cells express polyreactive, somatically mutated immunoglobulins. Blood 115, 581-591 (2010).

10. Mueller, A. et al. The role of antigenic drive and tumor-infiltrating accessory cells in the pathogenesis of Helicobacter-induced mucosa-associated lymphoid tissue lymphoma. The American journal of pathology 167 , 797-812 (2005).

11. Shiu, J., Czinn, S.J., Kobayashi, K.S., Sun, Y. \& Blanchard, T.G. IRAK-M expression limits dendritic cell activation and proinflammatory cytokine production in response to Helicobacter pylori. PloS one 8, e66914 (2013).

12. Sayi, A. et al. TLR-2-activated B cells suppress Helicobacter-induced preneoplastic gastric immunopathology by inducing T regulatory-1 cells. $\mathrm{J}$ Immunol 186, 878-890 (2011).

13. Sun, X. et al. TLR2 mediates Helicobacter pylori-induced tolerogenic immune response in mice. PloS one 8, e74595 (2013).

14. Rad, R. et al. Extracellular and intracellular pattern recognition receptors cooperate in the recognition of Helicobacter pylori. Gastroenterology 136 2247-2257 (2009).

15. Yamamoto, K. et al. Anti-CXCL13 antibody can inhibit the formation of gastric lymphoid follicles induced by Helicobacter infection. Mucosal immunology 7, 1244-1254 (2014).

16. Cua, D.J. \& Tato, C.M. Innate IL-17-producing cells: the sentinels of the immune system. Nature reviews. Immunology 10, 479-489 (2010).

17. Sonnenberg, G.F., Fouser, L.A. \& Artis, D. Border patrol: regulation of immunity, inflammation and tissue homeostasis at barrier surfaces by IL-22. Nature immunology 12, 383-390 (2011).

18. Lei, J. et al. Heterogeneity of the biological properties and gene expression profiles of murine bone marrow stromal cells. The international journal of biochemistry \& cell biology 45, 2431-2443 (2013).

19. Noverr, M.C., Noggle, R.M., Toews, G.B. \& Huffnagle, G.B. Role of antibiotics and fungal microbiota in driving pulmonary allergic responses. Infection and immunity 72, 4996-5003 (2004).
20. Gladiator, A., Wangler, N., Trautwein-Weidner, K. \& LeibundGutLandmann, S. Cutting edge: IL-17-secreting innate lymphoid cells are essential for host defense against fungal infection. J Immunol 190, 521-525 (2013).

21. Genta, R.M., Hamner, H.W. \& Graham, D.Y. Gastric lymphoid follicles in Helicobacter pylori infection: frequency, distribution, and response to triple therapy. Human pathology 24, 577-583 (1993).

22. Bayerdorffer, E. et al. Regression of primary gastric lymphoma of mucosaassociated lymphoid tissue type after cure of Helicobacter pylori infection. MALT Lymphoma Study Group. Lancet 345, 1591-1594 (1995).

23. Kobayashi, K. et al. IRAK-M is a negative regulator of Toll-like receptor signaling. Cell 110, 191-202 (2002).

24. Zhou, $\mathrm{H}$. et al. IRAK-M mediates Toll-like receptor/L-1R-induced NFkappaB activation and cytokine production. The EMBO journal 32, 583-596 (2013).

25. Biswas, A. et al. Negative regulation of Toll-like receptor signaling plays an essential role in homeostasis of the intestine. European journal of immunology 41, 182-194 (2011).

26. Mebius, R.E. Organogenesis of lymphoid tissues. Nature reviews. Immunology 3, 292-303 (2003).

27. Wolk, K. et al. IL-22 increases the innate immunity of tissues. Immunity 21, 241-254 (2004).

28. Wolk, K. et al. IL-22 regulates the expression of genes responsible for antimicrobial defense, cellular differentiation, and mobility in keratinocytes: a potential role in psoriasis. European journal of immunology 36, 13091323 (2006).

29. Zheng, Y. et al. Interleukin-22 mediates early host defense against attaching and effacing bacterial pathogens. Nature medicine 14, 282-289 (2008).

30. Sonnenberg, G.F., Monticelli, L.A., Elloso, M.M., Fouser, L.A. \& Artis, D. $\mathrm{CD} 4(+)$ lymphoid tissue-inducer cells promote innate immunity in the gut. Immunity 34, 122-134 (2011).

31. He, Q., Wang, J.P., Osato, M. \& Lachman, L.B. Real-time quantitative PCR for detection of Helicobacter pylori. Journal of clinical microbiology 40, 3720-3728 (2002).

32. Liu, H., Rahman, A., Semino-Mora, C., Doi, S.Q. \& Dubois, A. Specific and sensitive detection of $\mathrm{H}$. pylori in biological specimens by real-time RT-PCR and in situ hybridization. PloS one 3, e2689 (2008).

33. Kasai, M. et al. Use of quantitative real-time PCR to study the kinetics of extracellular DNA released from Candida albicans, with implications for diagnosis of invasive Candidiasis. Journal of clinical microbiology 44, 143-150 (2006).

34. Sonnenberg, G.F. et al. Innate lymphoid cells promote anatomical containment of lymphoid-resident commensal bacteria. Science 336 , 1321-1325 (2012).

35. Ravel, J. et al. Vaginal microbiome of reproductive-age women. Proceedings of the National Academy of Sciences of the United States of America 108 (Suppl 1), 4680-4687 (2011). 